\title{
Intravitreal Injection of Bevacizumab in a Case of Occlusive Retinal Vasculitis Accompanied by Syphilitic Intraocular Inflammation
}

\author{
Kan Ishijima ${ }^{a}$ Kenichi Namba $^{a}$ Shigeaki Ohno $^{b}$ \\ Kiyofumi Mochizuki ${ }^{c}$ Susumu Ishida ${ }^{a}$ \\ Departments of ${ }^{a}$ Ophthalmology and ${ }^{b}$ Ocular Inflammation and Immunology, \\ Hokkaido University Graduate School of Medicine, Sapporo, and \\ 'Department of Ophthalmology, Gifu University Graduate School of Medicine, \\ Gifu, Japan
}

\section{Key Words}

Bevacizumab - Occlusive retinal vasculitis - Syphilis · Jarisch-Herxheimer reactions . Treponema

\begin{abstract}
Background: We report a rare case of syphilitic intraocular inflammation with occlusive retinal vasculitis treated with intravitreal injection of bevacizumab (IVB) in addition to conventional therapy for syphilis.

Case: A 24-year-old woman who complained floaters in both her eyes showed occlusive retinal vasculitis OU. According to the high titer of the Treponema antigen and characteristic cutaneous eruption, she was diagnosed as secondary syphilis.

Observation: She was treated with oral amoxicillin and retinal photocoagulation OU. Then, administration of prednisolone was required to the intraocular inflammations considered as Jarisch-Herxheimer reactions. IVB was also performed toward exacerbated retinal neovascularization and showed transient effects.

Conclusion: We experienced a rare case of occlusive retinal vasculitis accompanied by syphilis intraocular inflammation. IVB was considered to be effective as an adjunctive therapy for inflammatory neovascularizations.
\end{abstract}




\section{Introduction}

Syphilis is one of the sexually transmitted diseases, and due to the educational instruction and development of antibiotics, the number of the syphilis patients has decreased markedly in the last half-century in Japan. According to the recent epidemiological survey, syphilis is in less than $1 \%$ the cause of intraocular inflammation in Japan (published in Japan). However, it is still an important disease and always should be kept in mind as one of the differential diagnoses. Syphilitic intraocular inflammation shows nonspecific ocular findings such as iridocyclitis, retinal vasculitis, and retinal pigment epithelium atrophy. However, occlusive retinal vasculitis as an ocular finding seen in syphilis is rare and so far only one case has been reported [1].

In this report, we show a rare case of occlusive retinal vasculitis accompanied by syphilis intraocular inflammation, in which intravitreal injection of bevacizumab (IVB; Roche, Basel, Switzerland) seemed to be effective for suppressing retinal neovascularization.

\section{Case Report}

A 24-year-old woman visited an ophthalmology clinic with the complaint of floaters in both eyes and was referred to Gifu University Hospital, Japan, in October 2007. Decimal visual acuity was 1.5 OU. No inflammatory cells were detected in the anterior chamber. Diffuse vitreous opacity, slight redness of optic disc and sheathing of retinal artery $\mathrm{OU}$, and retinal hemorrhage adjacent to retinal veins in the inferior quadrant $\mathrm{OD}$ and in the superior quadrant OS were seen. Fluorescein angiography showed non-perfusion area and dye leakage indicating retinal neovascularization (fig. 1).

QuantiFERON ${ }^{\circledR}$-TB assay for cellular immunity to Mycobacterium tuberculosis was performed, but the result was negative. Syphilis lipid antigen (rapid plasma reagin; RPR) and Treponema antigen (Treponema pallidum latex immunoassay; TPLA) showed high titer; 16 times and 2,280 TU, respectively. She showed characteristic cutaneous syphilis and was diagnosed as secondary syphilis.

After she moved to Hokkaido, Japan, she was immediately orally administered amoxicillin 1,500 $\mathrm{mg}$, and retinal photocoagulation was performed to the non-perfusion area OU. Oral amoxicillin was administered for 8 weeks, and TPLA decreased to 104.3 TU and has never risen again. However, anterior chamber cells and vitreous hemorrhages appeared OS and fluorescein angiography revealed increasing dye leakage from retinal vessels. Then, oral prednisolone $(40 \mathrm{mg} ; 0.7 \mathrm{mg} / \mathrm{kg} / \mathrm{day}$ ) was administered 4 weeks after antibiotics administration.

Despite these intensive therapies, the retinal neovascularization gradually exacerbated and fluorescence leakage from neovessels increased. And additional photocoagulation could not be performed due to repeated small vitreous hemorrhages. Therefore, IVB $1.25 \mathrm{mg}$ was performed OU on April 23 with the approval of the Ethics Committee of the University Hospital and the informed consent of the patient. On May 23, 4 weeks after IVB, fluorescence leakage from new vessels markedly decreased (fig. 2). On June 17, 8 weeks after IVB, due to the increase of fluorescence leakage from new vessels, a second IVB was performed OU.

Fluorescence leakage decreased afterwards, and finally the leakage disappeared. However, in August, severe vitreous hemorrhage occurred OS, and pars plana vitrectomy with endophotocoagulation was performed. After the operation, fluorescence leakage and intraocular inflammation have never been seen OS until now. Vitreous hemorrhage never occurred OD. Final visual acuity in November was 1.2 OD, and 1.0 OS. Prednisolone was administered with gradual decrease of the dose until February 2009. 


\section{Discussions}

As for therapy for occlusive retinal vasculitis, treatment for a primary disease should be aimed (in this case, syphilis), together with photocoagulation to the non-perfusion area, and corticosteroids to control the inflammation. We performed antibiotic treatment for syphilis, photocoagulation to the non-perfusion area and steroid medicine in this case. However, the activity of neovascularization was so high that repeated vitreous hemorrhages occurred. Therefore, we performed IVB as an adjunctive therapy.

It has been reported that high concentration of vascular endothelial growth factor (VEGF) is detected from the vitreous cavity in some retinal diseases [2], and intravitreal injection of bevacizumab, which is a recombinant humanized monoclonal antibody against VEGF, is effective for the diseases such as diabetic retinopathy, branch retinal vein occlusion and age-related macular degeneration [3]. In addition, there is a report describing the effectiveness of IVB for inflammatory ocular neovascularization [4], and in this case we obtained a similar effective result.

In this case, administration of antibiotics worsened the intraocular inflammations including exacerbation of neovascularization. This phenomenon seemed to be JarischHerxheimer reactions (JHR) [5]. JHR is a paradoxical worsening phenomenon after antibiotic therapy, and it is speculated to be caused by endotoxin released from dead organisms killed by antibiotics. Although prednisolone was administered toward JHR at the peak stage of the inflammation, this therapy was not effective enough to suppress retinal neovascularizations.

Although IVB has only transient effects and needs to be performed repeatedly, it is considered to be a useful tool as an adjunctive therapy for inflammatory neovascularizations with JHR, as far as JHR is also a transient phenomenon.

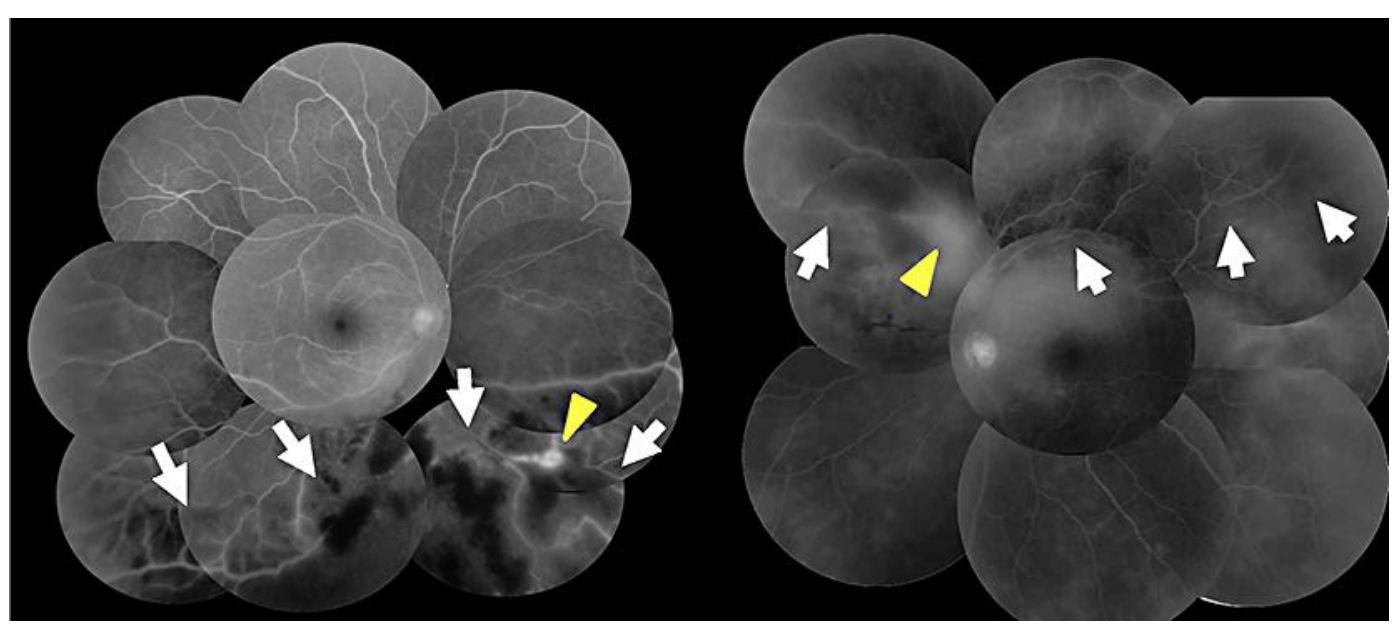

Fig. 1. Fluorescein angiography. Fluorescein angiography showed non-perfusion area (white arrows) and dye leakages (yellow arrows). 


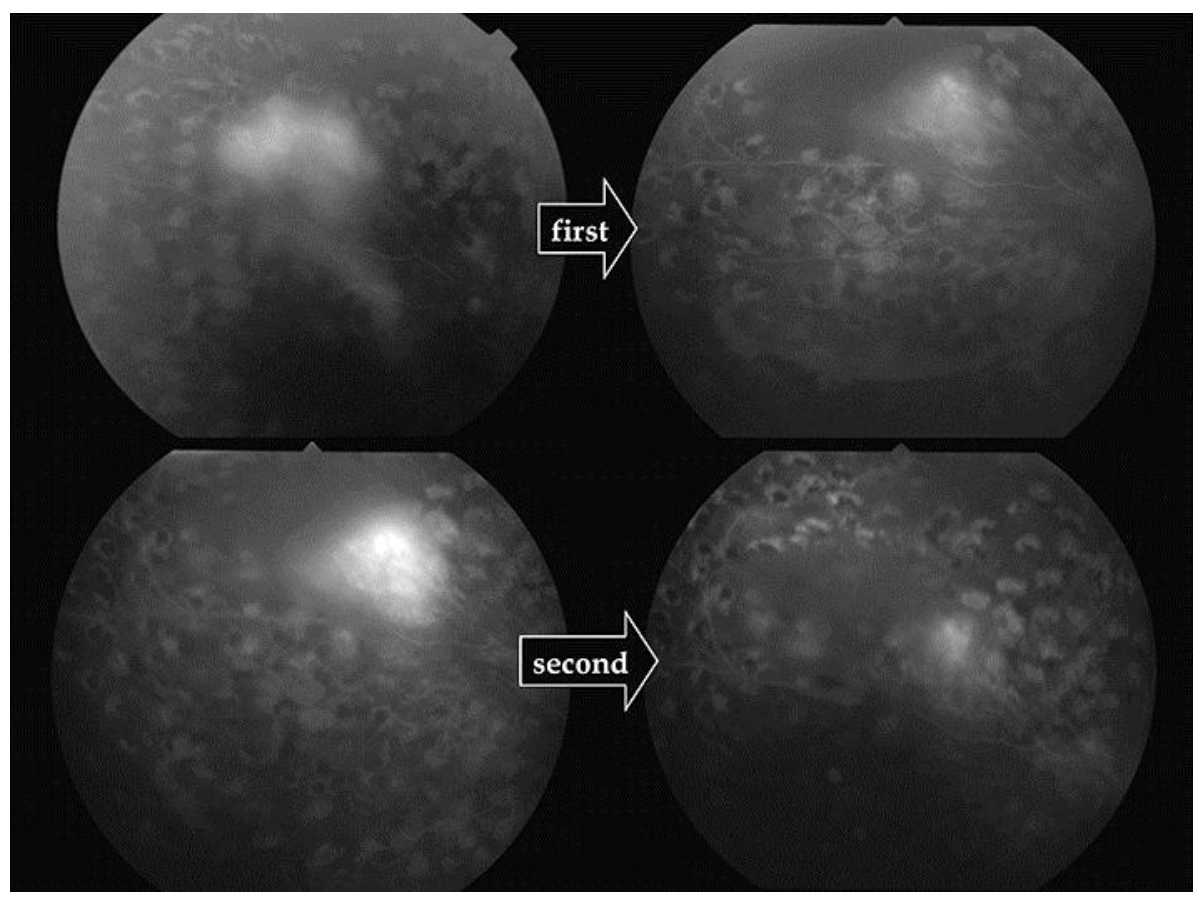

Fig. 2. Change of fluorescein angiography. After IVB, fluorescence leakage decreased markedly from retinal vessels (upper figures: first IVB, lower figures: second IVB).

\section{References}

1 Yokoi M, Kase M: Retinal vasculitis due to secondary syphilis. Jpn J Ophthalmol 2004;48:65-67.

-2 Gunther JB, Altaweel MM: Bevacizumab (Avastin) for the treatment of ocular disease. Surv Ophthalmol 2009;54:372-400.

-3 Aiello LP, Avery RL, Arrigg PG, Keyt BA, Jampel HD, Shah ST, Pasquale LR, Thieme H, Iwamoto MA, Park $\mathrm{JE}$, et al: Vascular endothelial growth factor in ocular fluid of patients with diabetic retinopathy and other retinal disorders. N Engl J Med 1994;331:1480-1487.

-4 Mansour AM, Mackensen F, Arevalo JF, Ziemssen F, Mahendradas P, Mehio-Sibai A, Hrisomalos N, Lai TY, Dodwell D, Chan WM, Ness T, Banker AS, Pai SA, Berrocal MH, Tohme R, Heiligenhaus A, Bashshur ZF, Khairallah M, Salem KM, Hrisomalos FN, Wood MH, Heriot W, Adan A, Kumar A, Lim L, Hall A, Becker M: Intravitreal bevacizumab in inflammatory ocular neovascularization. Am J Ophthalmol 2008;146:410416.

5 Cheung CM, Chee SP: Jarisch-Herxheimer reaction: paradoxical worsening of tuberculosis chorioretinitis following initiation of antituberculous therapy. Eye (Lond) 2009;23:1472-1473. 